\title{
Hydroxyethylstärke (HES): Neubewertung durch das Pharmacovigilance Risk Assessment Committee (PRAC)
}

\author{
PRAC Will Re-Examine its Recommendation Regarding Hydroxyethyl-Starch \\ (HES) Solutions
}

Autoren

Institute
K. P. Ittner ${ }^{1}$, B. Hossfeld ${ }^{2}$, J. Koppenberg ${ }^{3}$, P. Sefrin ${ }^{4}$

Die Institutsangaben sind am Ende des Beitrags gelistet.
Schlüsselwörter

- Notfallmedizin

- Pharmakotherapie

- Patientensicherheit

- Pharmakovigilanz

- Hydroxyethylstärke

\section{Keywords}

- emergency medicine

- drug therapy

- patient safety

- pharmacovigilance

- hydroxyethyl starch

\section{Bibliografie}

Dol http://dx.doi.org/ 10.1055/s-0033-1349462

Notarzt 2013; 29: 163-166

(c) Georg Thieme Verlag KG Stuttgart · New York

ISSN 0177-2309

Korrespondenzadresse

Priv.-Doz. Dr. med.

Karl Peter Ittner, DEAA, EDIC

Anästhesiologe, Klinischer

Pharmakologe, Klinik für

Anästhesiologie, Universitäts-

klinikum Regensburg

Franz Josef Strauss Allee 11

93042 Regensburg

Tel.: 0941/944-7801

Fax: 0941/944-7802

karl-peter.ittner@ukr.de

\section{Zusammenfassung \\ $\nabla$}

Der Ausschuss der Europäischen Arzneimittelagentur für Risikobewertung im Bereich der Pharmakovigilanz (PRAC) hat ein europäisches Risikobewertungsverfahren für die Anwendung hydroxyethylstärkehaltiger Infusionslösungen gestartet. Das BfArM empfiehlt deswegen bis zum Abschluss des europäischen Risikobewertungsverfahrens von der Anwendung hydroxyethylstärkehaltiger Infusionslösungen abzusehen, eine Anwendung ist aber noch nicht verboten. Bis zum Vorliegen der Ergebnisse des Bewertungsverfahrens ist der Einsatz von HES in jedem Einzelfall kritisch abzuwägen und auf Patienten mit akut vitalbedrohlichen, anderweitig nicht beherrschbaren Blut- und Volumenverlusten zu beschränken.

\section{Einführung}

$\nabla$

Das Bundesinstitut für Arzneimittel und Medizinprodukte (BfArM - http://www.bfarm.de) berichtete am 24.06.2013 auf seiner Internetseite „Risikoinformationen aus dem Jahr 2013“, dass der Ausschuss der Europäischen Arzneimittelagentur (EMA - www.ema.europa.eu/) für Risikobewertung im Bereich der Pharmakovigilanz (PRAC) in seiner Sitzung vom 10.-13. Juni 2013 festgestellt hat, dass der Nutzen von hydroxyethylstärkehaltigen Infusionslösungen die Risiken nicht länger überwiegt. Das PRAC empfahl deswegen ein Ruhen der entsprechenden Zulassungen.

Das BfArM entschloss sich aber für eine weniger strenge Lösung. Bis zum Abschluss des Verfahrens solle man von der Anwendung HES-haltiger Infusionslösungen absehen.

Nachdem pharmazeutische Unternehmen Anträge gegen das Ruhen der Zulassung gestellt haben, hat das PRAC in seiner Sitzung vom 10.-13. Juli 2013 ein Ruhen der Zulassungen zunächst zurückgestellt. Auf der DGAI-Homepage wurde eine

\section{Abstract \\ $\nabla$}

At the request of the German Federal Institute for Drugs and Medical Devices (BfArM), the EMA's Pharmacovigilance Risk Assessment Committee (PRAC) has initiated a review procedure regarding the basic evaluation of the benefit-risk balance of solutions for infusion containing hydroxyethyl starch (HES). Here we present an overview of facts to provide emergency physicians objective information.
Stellungnahme der Präsidenten der DGAI und des BDA sowie der Präsidentin der DAAF zu Hydroxyethylstärke am 18.07.2013 veröffentlicht. Mit dieser Information wollen wir Notärztinnen/Notärzte die wichtigsten Fakten übermitteln.

\section{Hintergrund $\nabla$}

In $\odot$ Tab. 1 sind alle wichtigen Hintergrundinformationen zusammengefasst.

\section{Woran kann man sich momentan zu HES orientieren? \\ $\nabla$}

Wir haben in $\bullet$ Tab. 2 die wichtigsten Informationen zusammengestellt.

Die momentan aktuellste, zusätzlich evidenzbasierte und praxisorientierte Darstellung zur notärztlichen Volumentherapie findet sich auf uptodate. Leider ist diese Datenbank kostenpflichtig. Für diejenigen, die sich diese Ausführungen nicht 
Tab. 1 Nutzen-Risikobewertung von HES, zeitliche Zusammenstellung der letzten Monate.

\begin{tabular}{|c|c|}
\hline Datum & Themen/Inhalte und Links \\
\hline 2012 & $\begin{array}{l}\text { Zwei hochkarätig publizierte Studien bei Intensivpatienten ergaben, dass HES im Vergleich zu Kristalloiden insbesondere eine } \\
\text { Nephrotoxizität verursachte: } \\
\text { 1. Perner A, Haase N, Guttormsen AB et al. Hydroxyethyl starch 130/0.42 versus Ringer's acetate in severe sepsis. The New England } \\
\text { journal of medicine 2012; } 367: 124-134 \\
\text { http://www.ncbi.nlm.nih.gov/pubmed/22738085 } \\
\text { 2. Myburgh JA, Finfer S, Bellomo R et al. Hydroxyethyl starch or saline for fluid resuscitation in intensive care. The New England journal } \\
\text { of medicine 2012; } 367: 1901-1911 \\
\text { http://www.ncbi.nlm.nih.gov/pubmed/23075127 }\end{array}$ \\
\hline ab Feb. 2013 & $\begin{array}{l}\text { Thematische Begleitung in „Deutsches Ärzteblatt“ } \\
\text { http://www.aerzteblatt.de/ } \\
\text { http://www.aerzteblatt.de/nachrichten/53484/Meta-Analysen-Hydroxyethylstaerke-erhoeht-Risiko-auf-Nierenversagen-und-Tod? } \\
\text { s=hes } \\
\text { http://www.aerzteblatt.de/nachrichten/54799/Hydroxyethylstaerke-EMA-fuer-Verbot-von-Volumenersatzmittel?s=hes } \\
\text { http://www.aerzteblatt.de/archiv/141430/Volumentherapie-mit-Hydroxyethylstaerke-beim-kritisch-Kranken-Eine- } \\
\text { Neubewertung?s=hes } \\
\text { http://www.aerzteblatt.de/archiv/141427/Umdenken-in-der-Volumentherapie-Nutzen-oder-Risiko-von-Hydroxyethylstaerke?s=hes }\end{array}$ \\
\hline März 2013 & $\begin{array}{l}\text { Die Europäische Arzneimittelagentur hat auf Antrag des BfArM ein Risikobewertungsverfahren zur grundlegenden Überprüfung des } \\
\text { Nutzen-Risiko-Verhältnisses von HES-haltigen Infusionslösungen eingeleitet. } \\
\text { http://www.bfarm.de/SharedDocs/1_Downloads/DE/BfArM/publ/bulletin/2013/1-2013.pdf?__blob=publicationFile } \\
\text { http://www.bfarm.de/DE/Pharmakovigilanz/risikoinfo/2013/RI-hes.html?nn=1694416 }\end{array}$ \\
\hline Frühjahr 2013 & $\begin{array}{l}\text { Pro-Con-Diskussion auf „INTENSIV-News“ } \\
\text { http://www.medicom.cc/medicom-de/inhalte/intensiv-news/intensiv-news.php } \\
\text { http://www.medicom.cc/medicom-de/inhalte/intensiv-news/entries/IN113/Pro-Nach-VISEP-CHEST-6S-und-Sind-synthetische- } \\
\text { Kolloide-noch-indiziert.php } \\
\text { http://www.medicom.cc/medicom-de/inhalte/intensiv-news/entries/IN213/Kommentar-zum-Beitrag-Sind-synthetische-Kolloide- } \\
\text { noch-indiziert.php } \\
\text { http://www.medicom.cc/medicom-de/inhalte/intensiv-news/entries/IN313/7-Ein-Plaedoyer-fuer-die-Ehrlichkeit.php }\end{array}$ \\
\hline 18.06 .2013 & $\begin{array}{l}\text { Das BfArM nimmt Stellung zu einer als „Rote-Hand-Brief“ deklarierten Mitteilung der Firma Serumwerke Bernburg } \\
\text { http://www.bfarm.de/DE/Pharmakovigilanz/risikoinfo/2013/Rl-rhb-hes.html }\end{array}$ \\
\hline 10. -13.06 .2013 & $\begin{array}{l}\text { Sitzung der PRAC; Empfehlung für ein Ruhen der HES-Zulassungen. } \\
\text { http://www.ema.europa.eu/ema/index.jsp?curl=pages/medicines/human/referrals/Hydroxyethyl_starch-containing_solutions/ } \\
\text { human_referral_prac_000012.jsp\&mid=WC0b01ac05805c516f }\end{array}$ \\
\hline 24.06 .2013 & $\begin{array}{l}\text { BfArM empfiehlt, von der Anwendung hydroxyethylstärkehaltiger Infusionslösungen abzusehen. } \\
\text { http://www.bfarm.de/DE/Pharmakovigilanz/risikoinfo/2013/RI-hes2.html }\end{array}$ \\
\hline 24.06 .2013 & $\begin{array}{l}\text { Die Food and Drug Administration (FDA) gibt eine „Black-Box-Warnung“ für HES heraus. } \\
\text { http://www.fda.gov/Safety/MedWatch/SafetyInformation/SafetyAlertsforHumanMedicalProducts/ucm358349.htm }\end{array}$ \\
\hline 08. -11.07 .2013 & $\begin{array}{l}\text { Sitzung der PRAC; Empfehlung der Sitzung vom 10. }-13.06 .2013 \text { wird ausgesetzt. } \\
\text { http://www.ema.europa.eu/ema/index.jsp?curl=pages/medicines/human/referrals/Hydroxyethyl_starch-containing_medicines/ } \\
\text { human_referral_prac_000029.jsp\&mid=WC0b01ac05805c516f }\end{array}$ \\
\hline 19.07.2013 & $\begin{array}{l}\text { PlasmaVolume Redibag® (Hydroxyethylstärke (HES)): Freiwilliger Rückruf des Arzneimittels. } \\
\text { http://www.bfarm.de/DE/Pharmakovigilanz/risikoinfo/2013/info-redibag.html } \\
\text { http://www.akdae.de/Arzneimittelsicherheit/Weitere/Archiv/2013/20130719.html }\end{array}$ \\
\hline
\end{tabular}

besorgen können, versuchen wir in der nächsten Nummer des „Notarztes“ diese in deutscher Sprache zur Verfügung zu stellen. Für das Volumenmanagement beim Polytrauma verweisen wir weiterhin auf die Ausführungen in der AWMF-Leitlinie (Link in - Tab.2).

Solange die Fachinformationen nicht durch „Rote Hand Briefe“ ersetzt sind, kann man sich bei der Maximaldosierung der einzelnen HES-Lösungen auf www.fachinfo.de informieren ( $\bullet$ Tab.2). Hilfreich wäre es aber, wenn seitens der pharmazeutischen Industrie, durch „Rote Hand Briefe“ rasch eine differenziertere Darstellung erfolgen könnte. Die momentane Indikation „Hypovolämie“ ohne weitere Unterscheidung zwischen absoluter und relativer Hypovolämie trägt auf keinen Fall zu einer sicheren Anwendung bei.

Die Präsidenten der DGAI und des BDA sowie die Präsidentin der DAAF haben eine gemeinsame Stellungnahme am 18.07.2013 veröffentlicht. Diese Empfehlungen zu Hydroxyethylstärke sollten in der Notfallmedizin beachtet werden ( Tab.2).

\section{Worauf man noch achten sollte}

$\nabla$

Im Notarztdienst wird HES/Hyperhes primär beim hämorrhagischen-hypovolämischen Schock eingesetzt. Neben HES stehen zur Kreislaufstabilisierung kristalline Lösungen und Katecholamine (insbesondere Noradrenalin) zur Verfügung. Nach der gemeinsamen Empfehlung der Fachgesellschaften ( $\bullet$ Tab.2) ist der Einsatz von HES in jedem Einzelfall kritisch abzuwägen und auf Patienten mit akut vitalbedrohlichen, anderweitig nicht beherrschbaren Blut- und Volumenverlusten zu beschränken. $\mathrm{Zu}$ „Hyperhes“ gibt es bisher noch keine Empfehlungen. Hyperhes ist vor allem eine hyperosmolare Salzlösung, deren Wirkung durch das Kolloid gesichert werden soll, wobei zu überlegen ist, ob diese primäre Wirkung nicht auch ohne HES erreicht werden kann.

Die Anwendung von HES beim Krankheitsbild des anaphylaktischen Schocks mit einer stadienabhängigen progredienten relativen Hypovolämie sollte man sich gut überlegen. Die Anaphylaxie ist nicht bei den Indikationen zu HES aufgeführt (off-label-use). 
Tab.2 Informationen zur Anwendung von HES-Infusionen.

\begin{tabular}{|c|c|}
\hline $\begin{array}{l}\text { Erscheinungs- } \\
\text { bzw. Zugriffsdaten }\end{array}$ & Link \\
\hline 1.07 .2011 & $\begin{array}{l}\text { Die AWMF-S3-Leitlinie Polytrauma/Schwerverletzten-Behandlung geht in Kapitel 1.3 (ab S. 39) sehr differenziert auf das Flüssig- } \\
\text { keits- und Volumenmanagement ein. Die Leitlinie hat ihre Gültigkeit bis zum 31.12.2014 } \\
\text { http://www.awmf.org/leitlinien/detail/II/012-019.html } \\
\text { http://www.awmf.org/uploads/tx_szleitlinien/012-019I_S3_Polytrauma_Schwerverletzten-Behandlung_2011-07.pdf }\end{array}$ \\
\hline 24.06 .2013 & $\begin{array}{l}\text { Das BfArM empfiehlt bis zum Abschluss des europäischen Risikobewertungsverfahrens von der Anwendung hydroxyethylstärke- } \\
\text { haltiger Infusionslösungen abzusehen (siehe Details in } \bullet \text { Tab. 1). } \\
\text { http://www.bfarm.de/DE/Pharmakovigilanz/risikoinfo/2013/RI-hes2.html }\end{array}$ \\
\hline 24.06 .2013 & $\begin{array}{l}\text { FDA-Empfehlung in der „Black-Box-Warnung“ für HES. } \\
\text { http://www.fda.gov/Safety/MedWatch/Safetylnformation/SafetyAlertsforHumanMedicalProducts/ucm } 358349 \text {.htm } \\
\text { Recommendations for Health Professionals include the following: } \\
\text { - Do not use HES solutions in critically ill adult patients including those with sepsis, and those admitted to the ICU. } \\
\text { - Avoid use in patients with pre-existing renal dysfunction. } \\
\text { - Discontinue use of HES at the first sign of renal injury. } \\
\text { - Need for renal replacement therapy has been reported up to } 90 \text { days after HES administration. Continue to monitor renal } \\
\text { function for at least } 90 \text { days in all patients. } \\
\text { - Avoid use in patients undergoing open heart surgery in association with cardiopulmonary bypass due to excess bleeding. } \\
\text { - Discontinue use of HES at the first sign of coagulopathy. }\end{array}$ \\
\hline 17.07 .2013 & $\begin{array}{l}\text { Nachdem die Zulassung von HES nicht zu ruhen braucht, gelten momentan eigentlich die in den Fachinformationen ausgeführten } \\
\text { Darlegungen zu den einzelnen HES-Infusionen (www.fachinfo.de). Nachdem (siehe unten zu „Rote Hand Briefe“) aber Informa- } \\
\text { tionen der Pharmaindustrie zu „vorsichtiger Anwendung“ kursieren (z. B. nicht bei Sepsis, ...), sollte man auf jeden Fall diese In- } \\
\text { formationen zusätzlich berücksichtigen. Auch die AWMF-Leitlinie zum Polytrauma (siehe in dieser Tabelle) rät zu einer sehr diffe- } \\
\text { renzierten Anwendung mit HES-Infusionen. }\end{array}$ \\
\hline 17.07 .2013 & $\begin{array}{l}\text { Sucht man bei „Aktuelle Rote-Hand-Briefe“ so finden sich dort keine „Rote-Hand-HES-Briefe“. } \\
\text { http://www.bfarm.de/DE/Pharmakovigilanz/risikoinfo/roteHBriefe/roteHandBriefe-node.html } \\
\text { Es ist bekannt, dass HES-herstellende Firmen an Krankenhaus-Apotheken Stellungnahmen zur vorsichtigeren Anwendung abge- } \\
\text { geben haben. Es wäre gut, wenn in entsprechender Abstimmung mit dem BfArM diese Informationen auch an Notärzte z. B. als } \\
\text { Rote Hand Briefe gehen würden. }\end{array}$ \\
\hline 17.07.2013 & $\begin{array}{l}\text { In der kostenpflichtigen Datenbank uptodate (http://www.uptodate.com/contents/search) wird das Thema aktuell, auf sehr } \\
\text { hohem und praktischen Niveau, evidenzbasiert präsentiert: } \\
\text { http://www.uptodate.com/contents/prehospital-care-of-the-adult-trauma-patient?source=see_link } \\
\text { http://www.uptodate.com/contents/initial-evaluation-and-management-of-shock-in-adult-trauma?source=see_link\&anchor= } \\
\text { H5\#H5 }\end{array}$ \\
\hline 18.07.2013 & $\begin{array}{l}\text { http://www.dgai.de/01_0start-aktuelles.htm } \\
\text { Stellungnahme der Präsidenten der DGAI und des BDA sowie der Präsidentin der DAAF zu Hydroxyethylstärke } \\
\text { http://www.dgai.de/aktuelles/AI_07-08-2013_Editorial.pdf } \\
\text { Auszüge: } \\
\text { - ...primären Einsatz von Kristalloiden empfiehlt und bei Verwendung von Kolloiden den Einsatz von Gelatine oder Human- } \\
\text { albumin nahelegt. } \\
\text { - ... Verwendung von HES-Lösungen mit einem mittleren Molekulargewicht von } 200 \mathrm{kDa} \text { (HES 200/0,5 und HES 200/0,62) } \\
\text { aufgrund des potenziellen Risikos von Nierenfunktionsstörungen für alle Indikationen auszusetzen sei. } \\
\text { - HES-Lösungen mit einem mittleren Molekulargewicht von } 130 \mathrm{kDa} \text { (HES } 130 / 0,4 \text { und HES } 130 / 0,42 \text { ) sollten bis zum Vorliegen } \\
\text { der abschließenden Risikobewertung in der intensivmedizinischen Therapie vorsorglich ebenfalls nicht zur Anwendung kommen. } \\
\text { - Bis zum Vorliegen dieser Ergebnisse empfehlen die Unterzeichner vorsorglich, den Einsatz von HES in jedem Einzelfall kritisch } \\
\text { abzuwägen und auf Patienten mit akut vitalbedrohlichen, anderweitig nicht beherrschbaren Blut- und Volumenverlusten zu } \\
\text { beschränken. }\end{array}$ \\
\hline
\end{tabular}

Wegen der relativen Hypovolämie, Bronchokonstriktion und einer Schrankenstörung (deswegen die Urtikaria) bedingt durch Histamin, müssen in erster Linie biochemisch sowohl H1-Rezeptoren (Antihistaminika), alpha-1-/und beta-2-Rezeptoren angegriffen werden (Adrenalin/B2-Mimetika) und unterstützend Kortikosteroide eingesetzt werden. Die ERC-Leitlinie warnt dezidiert vor einem HES-Einsatz bei Anaphylaxie [1].

Es ist unwahrscheinlich, dass wegen eines Hörsturzes ein Notarzt gerufen wird. Falls doch, kann die Entscheidung zu einer HESTherapie ohne Probleme durch den HNO-Facharzt in der Klinik getroffen werden.

\section{Schlussbemerkungen}

\section{$\nabla$}

Die momentane Diskussion zu HES macht uns Ärztinnen und Ärzte aus der Intensiv- und Notfallmedizin sicher auch sehr nach- denklich. Waren wir nicht alle mit dem Einsatz bei „allen Hypovolämien“ sehr zufrieden. Haben wir beim Einsatz von HES bei der Therapie des hypovolämischen Schocks auch an ein mögliches Nierenversagen durch „Zu viel“ HES gedacht? Bei der momentanen Lage ist bei Notfallpatienten der Einsatz von HES nur dann gerechtfertigt, wenn alle anderen Optionen ausgeschöpft sind und bei vitaler Bedrohung somit keine praktikablen Alternativen mehr vorhanden sind.

\section{Danksagung}

Wir danken Professor Dr. Dr. h.c. J. Schüttler (Direktor der Klinik für Anästhesiologie, Universitätsklinikum Erlangen) für die Durchsicht dieses Manuskripts.

\section{Interessenkonflikt}

$\nabla$

Die Autoren geben an, dass kein Interessenkonflikt besteht. 
Institute

Klinischer Pharmakologe des Universitätsklinikums Regensburg, Klinik für Anästhesiologie (Direktor: Prof. Dr. B. M. Graf, MSc.), Regensburg

Klinik für Anaesthesiologie \& Intensivmedizin (Direktor: Prof. Dr. med. L. Lampl), Sektion Notfallmedizin, Bundeswehrkrankenhaus Ulm, Ulm Abteilung für Anästhesiologie, Schmerztherapie und Rettungsmedizin Center da Sandà Engiadina Bassa (Direktor: Dr. Joachim Koppenberg), Scuol, Schweiz ${ }^{4}$ Vorsitzender der Arbeitsgemeinschaft der in Bayern tätigen Notärzte (agbn), stellv. Vorsitzender der Bundesvereinigung der Arbeitsgemeinschaften der Notärzte Deutschlands - BAND e. V., Würzburg

\section{Literatur}

1 Soar J, Perkins GD, Abbas G et al. Kreislaufstillstand unter besonderen Umständen: Elektrolytstörungen, Vergiftungen, Ertrinken, Unterkühlung, Hitzekrankheit, Asthma, Anaphylaxie, Herzchirurgie, Trauma, Schwangerschaft, Stromunfall. Notfall Rettungsmed 2010; 13: 698 701

\section{Buchbesprechung}

Präklinische Versorgung ausländischer Patienten - Eine Betrachtung der notärztlichen Situation

Machado C

Hamburg: Verlag Dr. Kovać; 2013: 309 Seiten, ISBN 978-3-8300-6676-7

Deutschland war und ist ein Imigrationsland. In den kommenden Jahren werden wir eine zunehmende Zahl älterer Menschen mit Migrationshintergrund haben. Durch steigendes Lebensalter erhöhen sich das Erkrankungs- und Verletzungsrisiko und damit die Wahrscheinlichkeit eines Notarzteinsatzes. Aus diesem Grund sollte eine Analyse der präklinischen Versorgung mit dem Schwerpunkt interkulturelle Kommunikation gemacht werden.

Das Buch ist in 9 Kapitel unterteilt. Im ersten Kapitel wird der geschichtliche Rahmen abgesteckt und die Gründe für die Einwanderung ausländischer Staatsangehöriger und eine Begriffsbestimmung der Begriffe Migrant und Menschen mit Migrationshintergrund vorgenommen. Hierzu gehören auch eine Darstellung der gesundheitlichen und wirtschaftlichen Situation, der Wohnsituation und des Bildungsstandes ausländischer Personen. Im 2. Kapitel werden kulturwissenschaftliche Grundbegriffe definiert, um auf die kulturellen Einflussfaktoren im Gesundheitswesen einzugehen. In Kapitel 3 wird der Begriff Kultur speziell unter dem Aspekt der interkulturellen Kommunikation dargelegt, um die Auswirkungen kultureller Einflussfaktoren auf die menschliche Lebenswelt zu erschließen. Unterschiedliches Gesundheits- und Krankheitsverständnis kann zu Konflikten und Missverständnissen führen mit daraus resultierenden Fehldiagnosen und Fehltherapien. Aufgrund der hohen Anzahl von Patienten muslimischem Glaubens wird ein Exkurs zur Darstellung grundsätzliche Glaubensinhalte des Islams mit den medizinisch relevanten Auswirkungen eingeschoben. Im 4. Kapitel wird die medizinische Ausbildung in Deutschland und die Fortbildung im Bereich Notfallmedizin dargelegt. Während sich dieses Kapitel definitorischer Klarstellungen und philosophischen Überlegungen auf 108 Seiten widmet, kommt im Kapitel 5 die Darstellung der Fragestellung und der Methodik der bundesweiten Notarztstudie in deutschen Ballungszentren zum Zuge, wobei es um die Frage geht, welche kulturellen Faktoren der Problematik in der Notfallmedizin zugrunde liegen und welche erfahrungsbasierten Handlungsoptionen im interkulturellen Einsatz von Notärzten ergriffen werden.

Die Ergebnisse (Kapitel 6) sind unterteilt in die beiden Bereiche Berufsbild Notfallmedizin und interkulturelle Kommunikation. $\mathrm{Zu}$ Beginn der Ergebnisse wird die notärztliche Tätigkeit aus der Sicht der Betroffenen umfassend beschrieben. Unverständlich für das Thema ist die umfangreiche Darstellung des Verhältnisses Notarzt und Rettungssanitäter (Einsatzbeziehungsmodell) ohne Beziehung zum Grundthema. Erst nach 34 Seiten wird auf das Grundthema der interkulturellen Kommunikation eingegangen. Hier werden z.B. die verschiedenen Formen der
Kommunikation beschrieben, ohne daraus konkrete Empfehlung für die Praxis abzuleiten. Die Ergebnisbefunde beruhen meist auf einzelnen Erfahrungen der Notärzte. Es fehlen nachvollziehbare Daten und Häufigkeiten und mögliche konkrete Bezugsgrößen. In der Diskussion (Kapitel 7) wird versucht die Erkenntnisse der Forschungsfragen zu prüfen, zu validieren und zu erklären, welchen Beitrag sie für die Praxis leisten können. Im Bereich Notfallmedizin wird beklagt, dass es keine ausreichende fächerdeckende Fehler- und Kritikkultur gäbe. Es fehlt jedoch - wie sonst bei jeder wissenschaftlichen Befassung - der dazu nötige Abgleich der Literatur. Die im Literaturverzeichnis aufgeführten Studien lassen sich aufgrund fehlender Kennzeichnungen dem Text nicht zuordnen. Es reicht der Hinweis nicht „wie in der Literatur beschrieben“. Als Ergebnis der interkulturellen Kommunikation wird festgestellt, dass diese von Notärzten als explizit nicht belastend empfunden wurde. Es werden folgende notärztliche Charakteristika abgeleitet:

- Der Notarzt benötigt explizites, kognitiv verfügbares Kulturwissen beispielsweise über Verhaltenserwartungen und -weisen an fremdkulturellen Orten.

- Weiterhin ist ein profundes Wissen über und Verständnis für unterschiedliche Krankheits-, Gesundheits- und Körperkonzepte vonnöten, um kulturelle Äußerungen verstehen und interpretieren zu können.

- Der Handelnde muss neben einer Vielzahl anderer Kompetenzen über eine ausgeprägte interkulturelle Handlungskompetenz verfügen.

- Neben der Beherrschung ärztlicher Fertigkeiten und eines profunden fachübergreifenden Wissens wird die Einhaltung sozialer Erziehungs- und Höflichkeitsnormen explizit betont.

Darüber hinaus wird gefordert, eine interkulturelle Schulung in die Weiterbildung der Zusatzbezeichnung Notfallmedizin aufzunehmen.

Bei dem Buch handelt es sich nicht um eine übliche Publikation aus dem Bereich der Notfallmedizin, sondern um die Publikation einer Doktorarbeit. Gut für das Verständnis ist der ausführliche Exkurs über den Islam, während ansonsten die Aussagen grundsätzlich mit Originalzitaten aus der Umfrage teilweise recht umfänglich unterlegt werden. Dadurch ist das Buch schwer zu lesen. Durch die ständigen Unterbrechungen und die Suche nach verwertbaren praktischen Informationen ist schwer, weshalb das Buch auch weniger als Lesebuch zu nutzen ist. Für den an diesem Thema speziell Interessierten, findet sich jedoch eine Vielzahl von Informationen.

P. Sefrin, Würzburg 